\title{
Growth and development of human adipose tissue during early gestation*
}

\author{
C.M. Poissonnet ${ }^{\mathrm{a}}$, A.R. Burdi ${ }^{\mathrm{b}}$ and F.L. Bookstein ${ }^{\mathrm{c}}$ \\ "Centre de Recherches de Biologie du Développement Foetal et Néonatal, Hôpital Port-Royal, Paris, France. \\ ${ }^{b}$ Department of Anatomy and Cell Biology, and Center for Human Growth and Development, University of \\ Michigan, and Center for Human Growth and Development and Department of Radiology, University of \\ Michigan, Ann Arbor, MI, U.S.A.
}

Accepted for publication 13 December 1982

\section{Summary}

805 normal-for-age human cmbryos and fetuses were used to study early prenatal fat development. The investigation included observations on stages of fat morphogenesis at the light microscopic level and computerized image analyses of fat lobule size and number. The buccal fat pad was selected as a model system for the analyses. Fat tissue differentiates between the 14th and the 16th weeks: there are five morphogenic phases in adipose tissue formation, strongly associated with the formation of blood vessels. Fat lobules are the earliest structures to be identified before typical vacuolated fat cells appear. Concerning fat lobule size and number, we show that after the 23rd week the total number of fat lobules remains approximately constant, while from the 23rd to 29th week the growth of adipose tissue is determined mainly by an increase in size of the lobules. These results suggest that the 14th through the $23 \mathrm{rd}$ week is a sensitive period in fat lobule development, and that disturbances of normal adipogenesis during this period may play a role in the etiology of obesity in later life.

human adipose tissue; prenatal growth and development

\section{Introduction}

There is no precise description of prenatal morphogenesis of human subcutaneous fat despite an increasing number of related publications. Some studies deal with

\footnotetext{
* This work has been approved by the ethical committee of the University of Michigan Medical Center. Consent of the parents has been legally required for the submission of the fetuses to the Teratology Unit. Address for correspondence: Dr. C.M. Poissonnet, Centre de Recherches de Biologie du Développement Foetal et Néonatal, Hôpital Port-Royal, 123, Bd. de Port-Royal, 75014 Paris, France.
} 
early origins and the histogenesis of adipose tissue $[5,7,13,16,17]$, but they are not recent, and at the present time, information remains incomplete. In addition, quantitative data on fat cell size and number during gestation are very limited.

This study indicates that, in man, fat differentiation and proliferation occur during the second trimester of pregnancy. Before this period, no fat structures can be distinguished at the light microscopic level. The first part of this paper describes the key stages of fat formation (adipogenesis) based on histological data using light microscopy. In the second part, we present the results of a quantitative analysis of fat lobule size and number in the buccal fat pad from the 16th to 29 th week of gestation.

\section{Material and Methods}

Data were obtained from 805 human fetuses of both sexes from the Patten Embryology Research Collection of the University of Michigan. Specimens were considered normal when they conformed with published standards $[1,8,12]$ and were not associated with maternal pathology. Specimens were fixed in $10 \%$ formalin and subsequently prepared using standard histological procedures. Serial sections at 5 , 10 , or $15 \mu \mathrm{m}$ were stained with Masson trichrome (connective tissue stain) which

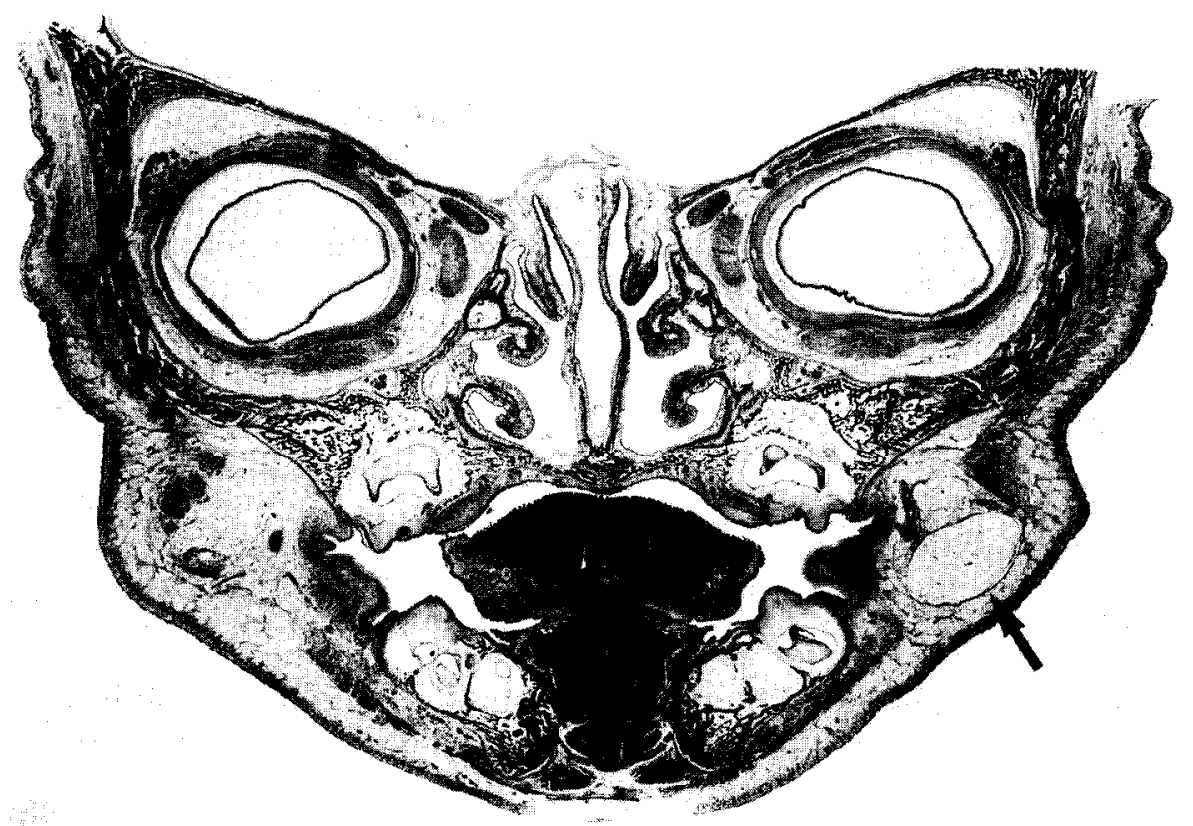

Fig. 1. Low-power photomicrograph of a frontal section of the face of a 28 -week-old fetus ( $253 \mathrm{~mm} \mathrm{CRL}$ length). Note that the buccal fat pad in the left cheek region (arrow) is well delineated. Staining in this and subsequent figures is with Masson trichrome stain. 
demonstrated preadipose tissue, and cell outlines and nuclei of definitive fat cells.

In all the serial sections from the 805 specimens, it was verified that no fat tissue was present before the age of 14 weeks. Patterns of adipogenesis were studied in 245 head specimens, in the cheeks, the chin, and the ocular fat pad. The buccal pad was chosen as a model of adipose tissue development because it is relatively large and forms early.

From the 245 sectioned heads ranging from 5 to 29 weeks, 32 specimens were selected. Fat lobule numbers and sizes were analyzed in these 32 specimens at the same buccal fat pad level. Fat lobules were examined rather than fat cells because the lobules were identifiable earlier, before typical vacuolated fat cells appear. Data were taken from 2, 3, or 4 left frontal cheek sections passing through the posterior half of the upper second molar (Fig. 1). The cheek was defined as the area formed on either side of the narrowed mouth between 9 and 11 weeks [9]. The sections were projected through a microscope onto a rectangular measurement window $(27 \times 35$ $\mathrm{cm}$ ), using a constant magnification $\times 100$. In each window, the individual fat lobules were outlined and digitized (using a Summagraphics digitizer) for subsequent statistical processing. A total of 2300 lobule outlines from 77 windows on the 32 specimens were analyzed. From the digitized lobule information, area and perimeter of each lobule outline, and mean perimeter and mean area of all lobules were statistically analyzed on a section-by-section basis. Lobule size was defined as to be equal to the mean intercept length (MIL) employed in stereology: MIL is the mean of lengths intercepted by the outline along random penetration of the form by a straight line. MIL was estimated from Tomkeieff's equation, as the ratio of mean perimeter length to mean area [14]. This calculation is appropriate to structures which, like fat lobules, are irregular in shape. This procedure differs from those used to determine fat cell size where the assumption is made that fat cells are spherical $[4,6]$.

\section{Results}

In the human face, fat tissue is distributed in the cheeks, the chin, and in the periocular region.

\section{Morphogenic sequence of fat formation}

Although adipose tissue, like other tissues, develops as a continuous process, prenatal fat formation can be divided, for convenience, into five morphogenic phases (Plate 1, Figs. 2-6).

Prior to the fourteenth prenatal week, future fat consists of loose connective tissue composed of an amorphous ground substance and fibres (Stage 1; Fig. 2). The first indication of adipogenesis is the aggregation of a dense mass of mesenchymal cells. Condensation of mesenchyme, which marks the end of the undifferentiated stage is asssociated with the proliferation of primitive vessels and indicates the emergence of characteristic fat tissue (Stage 2; Fig. 3). During this stage, from each small vessel a rich capillary network develops, around which mesenchymal cells differentiate into 
stellate preadipocytes. These mesenchymal lobules, which do not contain lipid droplets at this stage, are organized within a vascular structure or glomerulus, and will ultimately form a definitive fat lobule (Stage 3; Fig. 4). Development of early fat cells within the mesenchymal lobules coincides with further development of the vascular network. Thus, stages 2,3 and 4 overlap. Within the mesenchymal lobules, fine fat vacuoles form in the cytoplasm and increase in number. At this time, the primitive fat lobule includes (1) the vascular network or glomerulus, and (2) densely-packed vacuolated fat cells adjacent to small vessels (Stage 4; Fig. 5). In the final stage, definitive fat lobules are surrounded by perilobular mesenchyme. This condenses rapidly, and progressively thickens to form interlobular septa (Stage 5; Fig. 6).

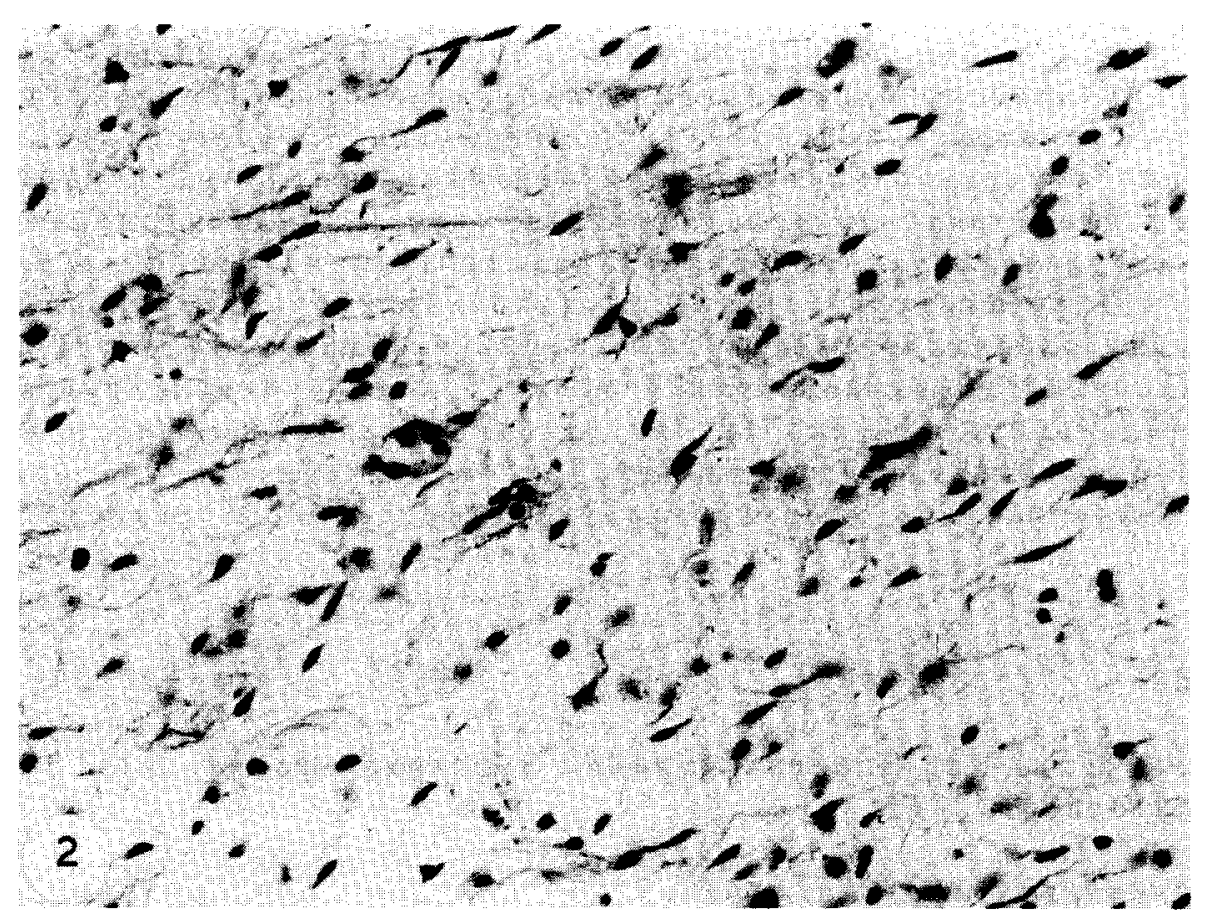

Figs. 2-6. Stages in the development of adipose tissue in the human buccal fat pad. (2) Stage 1: Undifferentiated fat tissue (without condensation). At 14 weeks ( $111 \mathrm{~mm} \mathrm{CRL}$ ), the cheek is filled with loose connective tissue, composed chiefly of stellate cells and ground substance $(\times 326)$. (3) Stage 2: Vascular invasion or angiogenesis. By 14.5 weeks, blood vessels proliferate and signal areas where early fat cells will organize in groups (arrows) $(\times 107)$. (4) Stage 3: Development of mesenchymal lobules. Note that this mesenchymal lobule (arrows) contains stellate mesenchymal cells or pre-adipocytes without lipid droplets. (19 weeks; $160 \mathrm{~mm} \mathrm{CRL)} \mathrm{(} \times 530)$. (5) Stage 4: Appearance of primitive fat lobules. Fat storage is visible around the 16th week. The vascular stalk (arrows) of each of these primitive fat lobules branches from one main vessel to enclose the densely packed fat cells (22 weeks; $180 \mathrm{~mm} \mathrm{CRL})(\times 677)$. (6) Stage 5: High magnification of definitive fat lobules at 28 weeks. Note that fat lobules are well separated from each other by connective tissue partitions or septa (arrows). ( $253 \mathrm{~mm} \mathrm{CRL)}(\times 100)$. 

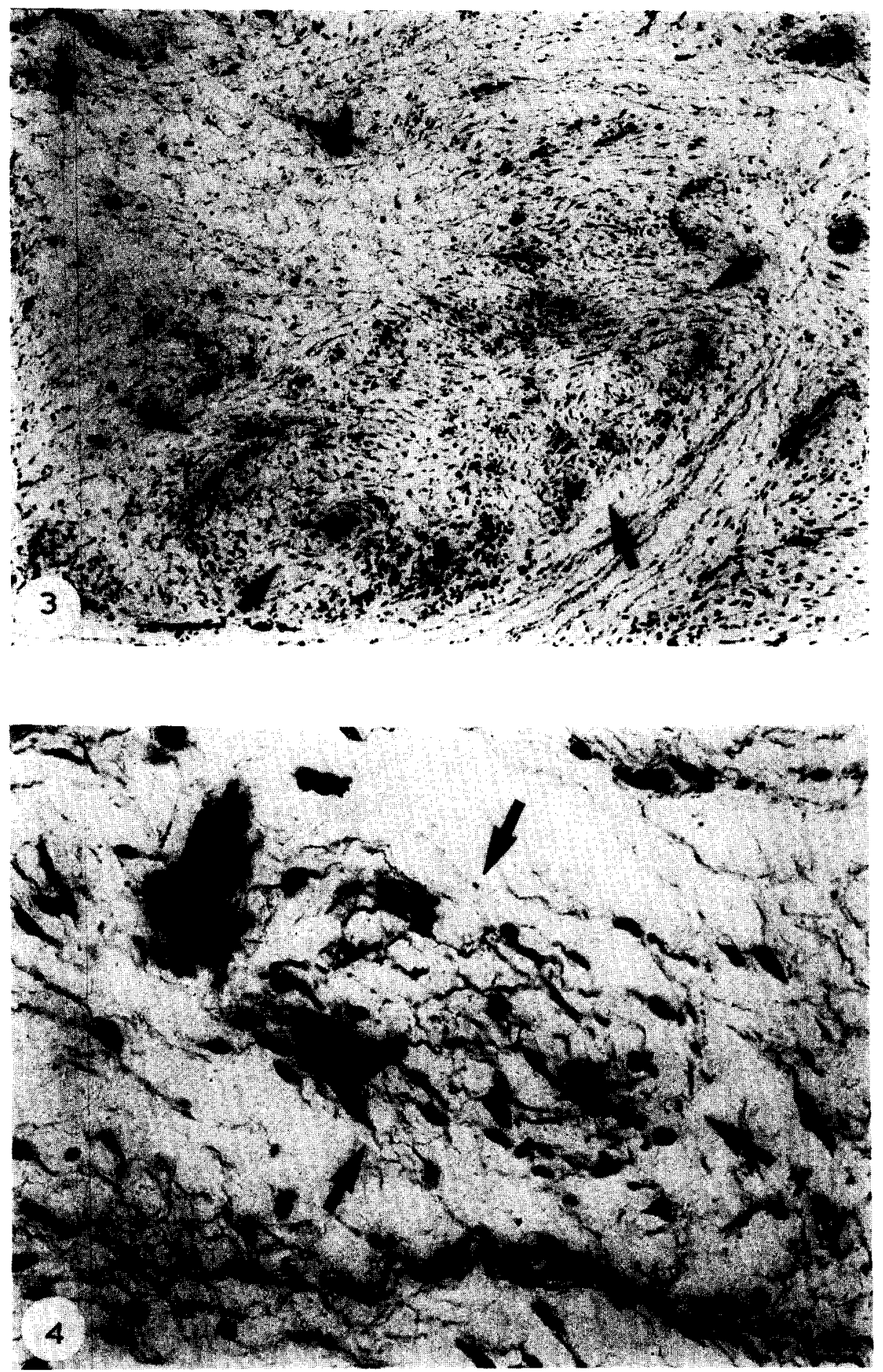

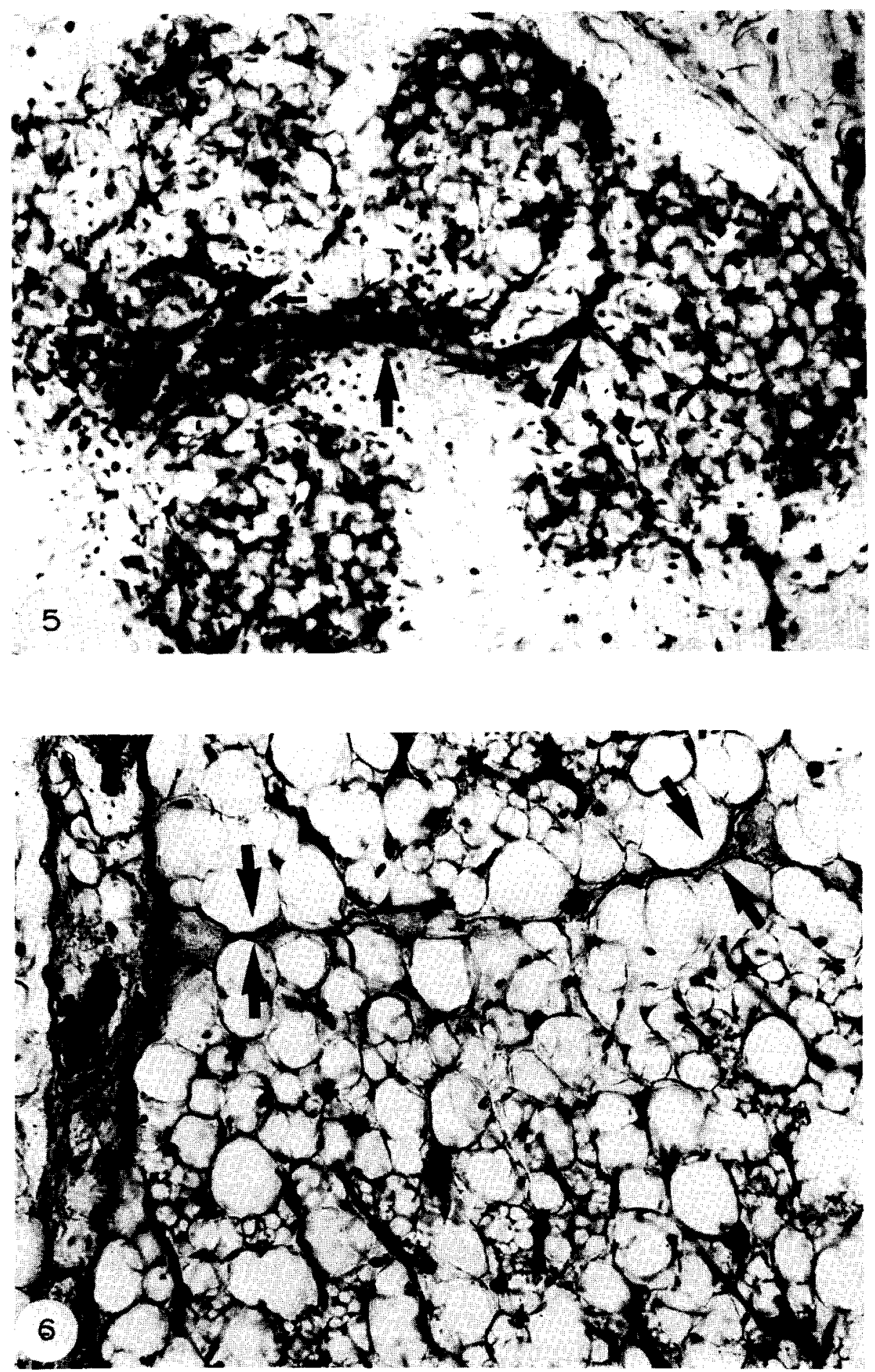
The formation of fat lobules or lobulogenesis (Fig. 7) begins at the end of the first stage and includes stages 2, 3, 4, and 5 of adipogenesis.

\section{Cheeks and chin}

The cheek is the first region where adipose tissue appears in the face after the fourteenth prenatal week. The development of adipose tissue progresses from the inner to the outer part of the cheek, according to a centrifugal gradient of maturation. Three types of lobules can be defined. These include primary, secondary and tertiary lobules, depending on where they are formed. Each of them follows the same morphogenic sequence of development as described above. Primary lobules: early in the fifteenth intrauterine week, primitive fat structures can be seen in the deep-seated area of the cheek and are already differentiated into primitive fat lobules (Stage 4). These primary lobules are sparsely located in superficial soft tissue below reticular dermis, extending into underlying connective tissue. They will later form the deep-seated buccal fat pad. Secondary lobules: towards the beginning of the seventeenth week, other lobules appear superficially in the subcutaneous tissue. There is an increase in the number of secondary lobules, leading to the formation of a thick continuous layer of fat that covers the midface from the upper part of the cheek to the chin. This more superficial covering constitutes the fat of the subcutis. Tertiary lobules: the last area where fat develops in the cheek is the peribulbar region of hair follicles in reticular dermis, the so-called papillae of the subcutis - an intradermal extension of the fat of the subcutis. Tertiary lobules are located deep around the follicles.

\section{Ocular fat pad}

Fat lobules develop after the eighteenth week around the optic vesicle, in similar

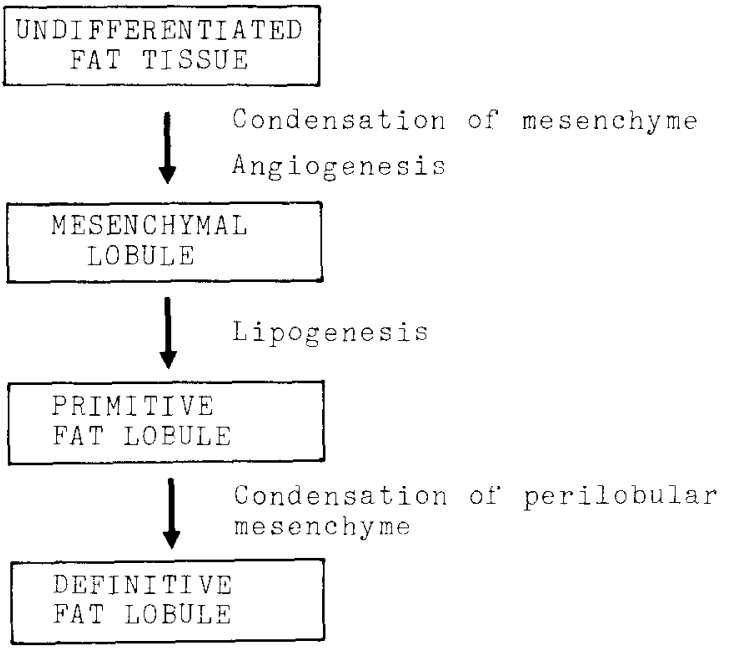

Fig. 7. Schematic representation of lobulogenesis. 
fashion as in the cheek. They are situated between the sclera and the bony wall of the orbit.

\section{Morphometric analysis}

Analysis of fat lobule size (Fig. 8) shows a linear relationship $(r=0.882)$ between the MIL-measured lobule size and crown-rump length (CRL). The relation between the number of lobules per $\mathrm{mm}^{2}$ (fat lobule density) and CRL (Fig. 9) is not linear, but rather indicates a leveling-off after about 23 weeks: for a CRL of $210-253 \mathrm{~mm}$ (corresponding to an age of 23-29 weeks) the smoothed number of fat lobules per $\mathrm{mm}^{2}$ probably does not increase. But the continuing increase observed in mean area over this size range is nevertheless reflected in an increase of total sectioned area covered by fat (Fig. 10).

\section{Discussion}

While adipose cells are relatively well described [6,11] in the literature, the ontogenesis of fat lobules remains unclear. This study shows five time-linked stages in fat tissue formation from the earliest appearance of a mesenchymal structure to the differentiation into the definitive fat lobule. While our observations are at the resolution of the light microscope, it appears that the first histological signs of fat tissue are linked with angiogenesis. This confirms the observations that fat cells cannot develop without vascularization $[10,17]$.

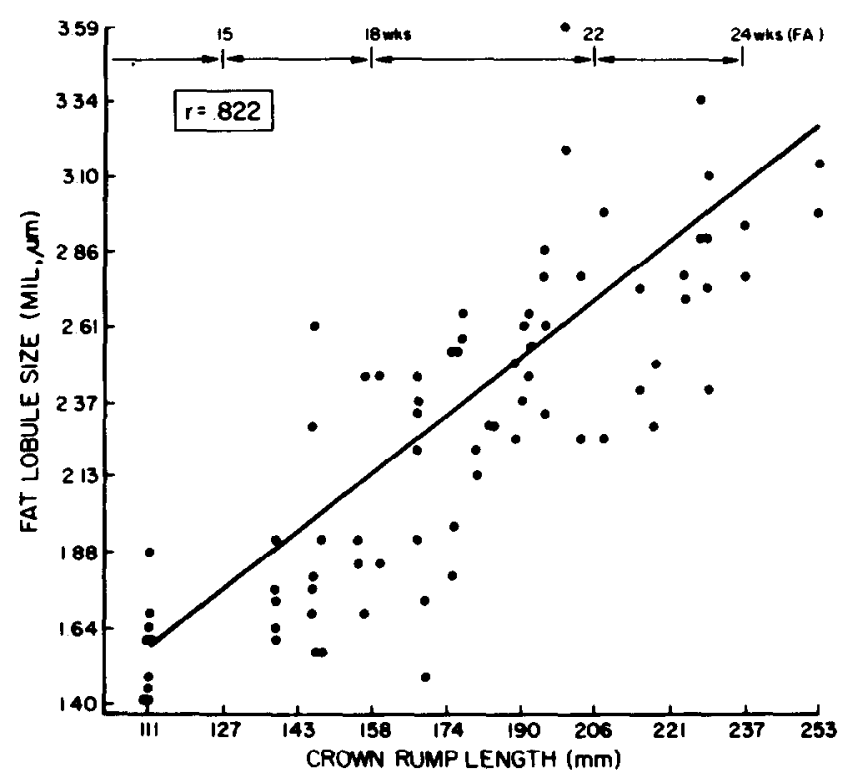

Fig. 8. Fat lobule size (mean intercept length, MIL) vs. crown-rump length (CRL). The solid line is the linear regression of MIL on CRL. 


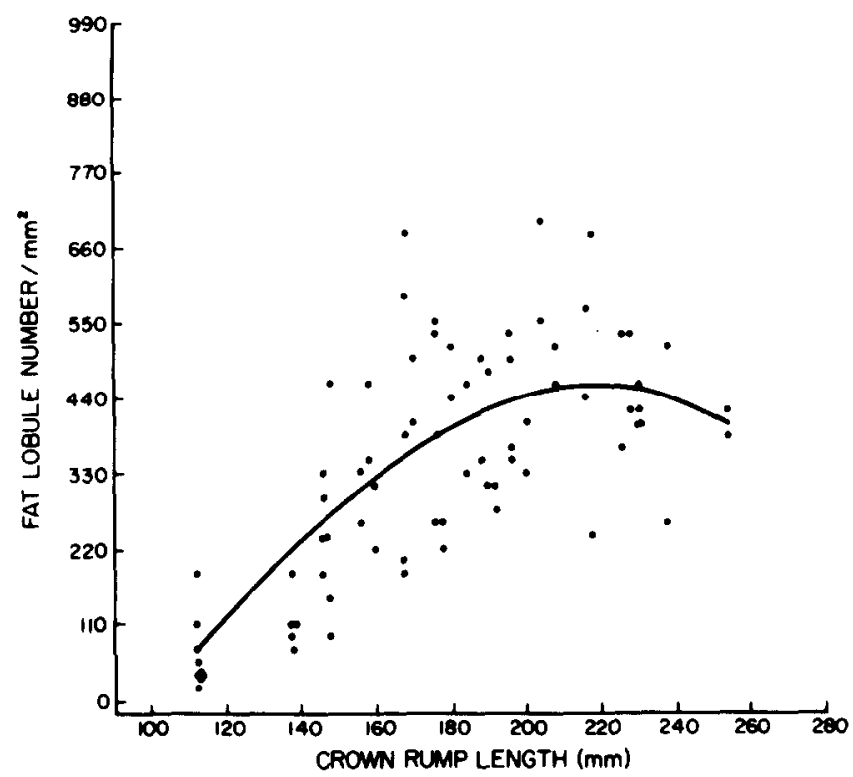

Fig. 9. Fat lobule number vs. crown-rump length. The solid curve is the quadratic regression of number on CRL.

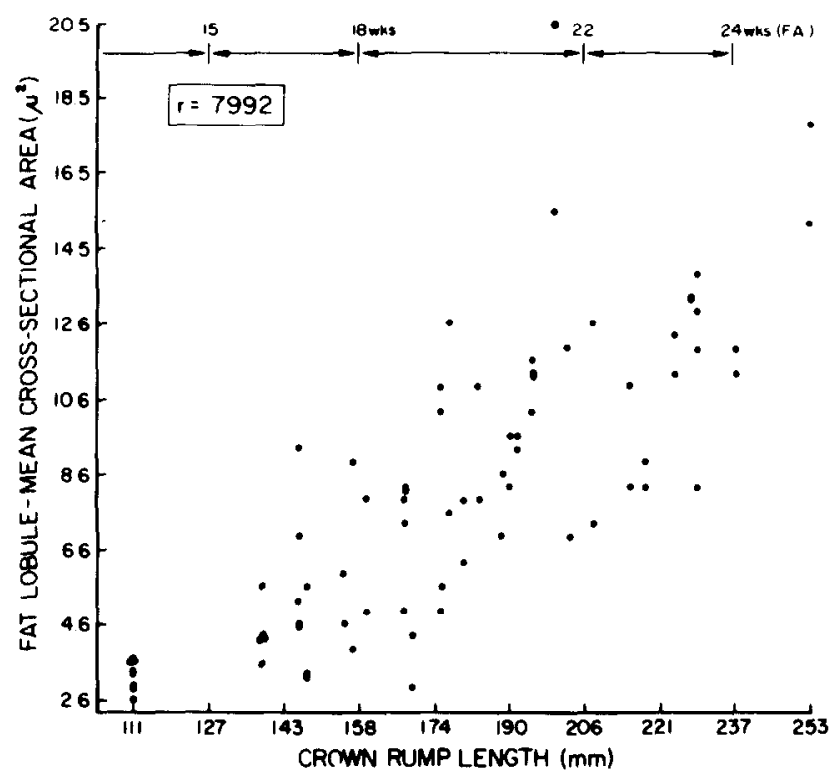

Fig. 10. Fat lobule, mean cross-sectional area vs. crown-rump length (CRL). 
Despite a considerable number of publications on fat, there is still no convincing study on the time of emergence of human fat structures. For Valdès-Dapena [15], deposits of fat form from the 17 th to the 20 th week of intrauterine life. Other reports state that adipose tissue appears later, between the 26 th and the 30 th weeks [11] or at about 30 weeks [3]. In fact, there is no reliable supportive evidence for these assumptions. Our findings indicate that mesenchymal matrix organization and formation of fat take place mostly during the second third of pregnancy, initially in the buccal fat pad soon after the 14th week. Later, fat tissue appears in the limbs and retroperitoneal areas. As demonstrated in the human face, fat differentiation occurs between the 14th and the 16th weeks. This rapid phase of fat formation is then followed by a period of intense fat-cell proliferation until the 23 rd week. Thus, fat cells are detectable much earlier than at a gestational age of 28 weeks, as previously suggested [2].

As regards fat lobule size and number, with this method of morphometry, the determination of fat lobule number does not represent the real number of lobules in each section. The lobules are more densely packed and become more irregular in shape when the fetuses are older. This may result in an over-estimation of lobules when only using the number of cross-sectional areas. Despite this bias, these results show that after the 23rd week the total number of fat lobules remains approximately constant. From the 23 rd to the 29 th week, the growth of adipose tissue is mainly due to an increase in size of the fat lobules, with development of their two components: the capillary network and the adipocytes.

In conclusion, this study indicates that in man the initiation of fat formation takes place between the 14th through 23 rd week of gestation. From our observations, there is an apparent morphogenic link between the onset of vascularization and the first appearance of adipose cells. Disturbances during this time may play a role in the etiology of obesity in later life.

\section{Acknowledgements}

Authors wish to acknowledge Drs. Stanley M. Garn and John T. Headington for their expert advice on this project, and Robert L. Wainright for his advice on the statistical procedures. This research was supported by the Kroc foundation (U.S.A.).

\section{References}

1 Arey, L.B. (1925): Simple formulae for estimating the age and size of human embryos. Anat. Rec., 30 , 389.

2 Boulton, T.J.C., Dunlop, M. and Court, J.M. (1974): Adipocyte growth in the first two years of life. Aust, Pediat. J., 10, 301-305.

3 Brook. C.G. (1972): Evidence for a sensitive period in adipose-cell replication in man. Lancet, 2 , 624-627.

4 Di Girolamo, M., Mendlinger, S. and Fertig, J.W. (1971): A simple method to determine fat cell size and number in four mammalian species. Am. J. Phys., 221, 3, 850-858. 
5 Flemming. W. (1871): On the formation and regression of fat cells in connective tissue with comment on the structure of the latter. Arch. R. Mikr. Anat., 7. 32.

6 Gurr. M.I. and Kirtland. J. (1978): Adipose tissue cellularity: a review. 1) Techniques for studying cellularity. Int. J. Obes., 2, 4, 401-427.

7 Hammer, J.A. (1895): Contribution to our knowledge of adipose tissue. Arch. Mikr. Anat., 45, 512.

8 O'Rahilly, R. (1973): Developmental stages in human embryos, including a survey of the Carnegie Collection, Carnegie Institution of Washington (Public. 631). Washington, D.C.

9 Remnick. H. (1970): Embryology of the face and the oral cavity. Fairleigh Dickinson University Press, East Brunswick. New Jersey.

10 Roth, J., Greenwood. M.R.C. and Johnson, P.R. (1981): The regenerating of facial sheaths in lipectomized Osborne-Mendel rats: morphological and biochemical indices of adipocyte differentiation and proliferation. Int. J. Obes., 5, 2, $131 \cdot 143$.

11 Simon. G. (1965): Histogenesis. In: Handbook of Physiology: adipose tissue, sec. 5. pp. 101-123. Editors: Reynold, A.E. and Cahill, G.F., Jr. Am. Phys. Soc.. Washington. D.C.

12 Streeter, G.L. (1921): Weight, sitting height. head size, foot length and menstrual age of human embryo. Carnegie Institution of Washington (Publication 274), Washington, D.C.

13 Toldt, C. (1870): Contribution to the histology and physiology of adipose tissue. Sitzungsber. Akad. Wiss. Wien Math. Naturwiss. K1., 62, 445.

14 Underwood, E.E. (1970): Quantitative Stereology. Addison-Wesley, Reading. Massachusetts.

15 Valdès-Dapena, M.A. (1979): Histology of the fetus and newborn. W.B. Saunders Company, Philadelphia, PA.

16 Wassermann, F. (1926): The fat organs of man: development structure and systematic place of the so-called adipose tissue. 7. Zellforsch. Mikr. Anat. Abt. Histochem.. 3. 325.

17 Wassermann. F. (1965): The development of adipose tissue. In: Handbook of Physiology: adipose tissue, sec. 5. pp. 87-100. Editors: Reynold. A.E. and Cahill, G.F., Jr. Am. Phys. Soc., Washington. D.C. 